\title{
Anomalous Convergence of a Continued Fraction for Ratios of Kummer Functions*
}

\author{
By Walter Gautschi
}

\begin{abstract}
We exhibit a phenomenon of apparent convergence to the wrong limit in connection with a continued fraction of Perron for ratios of Kummer functions. The phenomenon is further illustrated in the special cases of Bessel functions and incomplete gamma functions.
\end{abstract}

1. Introduction. From the differential equation satisfied by Kummer's function

$$
M(a, b ; z)=1+\frac{a}{b} \frac{z}{1 !}+\frac{a(a+1)}{b(b+1)} \frac{z^{2}}{2 !}+\ldots,
$$

Perron [4, p. 278] develops the following continued fraction,

$$
\frac{z M^{\prime}(a, b ; z)}{M(a, b ; z)}=\frac{a z}{b-z+} \frac{(a+1) z}{b+1-z+} \quad \frac{(a+2) z}{b+2-z+} \ldots, \quad b \neq 0,-1,-2, \ldots,
$$

where $M^{\prime}(a, b ; z)=(d / d z) M(a, b ; z)=(a / b) M(a+1, b+1 ; z)$. While the continued fraction converges for any complex $z$ not a zero of $M(a, b ; z)$, the convergence behavior can be extremely deceptive, when $|z| \gg \max (|a|,|b|)$, particularly if $\operatorname{Re} z>0$. The point is illustrated by concrete examples involving Bessel and incomplete gamma functions.

2. The Phenomenon of Apparent Convergence to the Wrong Limit. We assume, for simplicity, that $a \neq 0$ and $b-z \neq 0,-1,-2, \ldots$ Equation (1.1) can then be written in the form

$$
\frac{b-z}{a} \frac{M^{\prime}(a, b ; z)}{M(a, b ; z)}=\frac{1}{1+} \frac{a_{1}}{1+} \frac{a_{2}}{1+} \ldots
$$

where

$$
a_{k}=\frac{(a+k) z}{(b-z+k-1)(b-z+k)}, \quad k=1,2,3, \ldots
$$

Alternatively (cf., e.g., Wall [6, p. 17ff]),

$$
\frac{b-z}{a} \frac{M^{\prime}(a, b ; z)}{M(a, b ; z)}=\sum_{k=0}^{\infty} p_{k},
$$

where

$$
p_{0}=1, \quad p_{k}=\rho_{1} \rho_{2} \cdots \rho_{k}, \quad k=1,2,3, \ldots,
$$

Received January 20, 1977.

AMS (MOS) subject classifications (1970). Primary 33A30, 33A40, 40A1 5, 65D20.

Key words and phrases. Continued fractions, apparent convergence to the wrong limit, Bessel functions, incomplete gamma functions.

${ }^{*}$ Sponsored by the United States Army under Contract No. DAAG29-75-C-0024 and The National Science Foundation under grant MCS 76-00842. 
and

$$
\rho_{0}=0, \quad \rho_{k}=\frac{-a_{k}\left(1+\rho_{k-1}\right)}{1+a_{k}\left(1+\rho_{k-1}\right)}, \quad k=1,2,3, \ldots .
$$

The infinite series in (2.3) represents the continued fraction in (2.1) in the sense that the $n$th partial sum of the former is equal to the $n$th convergent of the latter, $n=$ $1,2,3, \ldots$.

Evidently, the terms $p_{k}$ in the series of (2.3) decrease or increase in absolute value according as $\left|\rho_{k}\right|<1$ or $\left|\rho_{k}\right|>1$, respectively. It is useful, therefore, to examine the behavior of $\left|\rho_{k}\right|$ as a function of $k$.

Assuming $\left|\rho_{k-1}\right|<1$, then $\left|\rho_{k}\right|<1$ certainly if $\left|a_{k}\right| \leqslant 1 / 4$. On the other hand, by (2.2), if $|z|>|b|+k$, then $\left|a_{k}\right|<(|a|+k)|z|(|z|-|b|-k)^{-2}$, and an elementary calculation shows the upper bound for $\left|a_{k}\right|$ to be $\leqslant 1 / 4$ if

$$
|z| \geqslant 2(|b|+2|a|+3 k)
$$

It follows that (2.5), together with $\left|\rho_{k-1}\right|<1$, implies $\left|\rho_{k}\right|<1$. Since, initially, $\rho_{0}=$ 0 , we obtain by induction that $\left|\rho_{k}\right|<1$ for all $k$ satisfying (2.5).

If $|z|$ is large, we see that the terms $p_{k}$ in (2.3) must decrease initially, the rate of decrease being greater the larger $|z|$. The continued fraction in (2.1) then gives the appearance of converging rapidly to a value of the order of magnitude 1 , yielding for $M^{\prime}(a, b ; z) / M(a, b ; z)$ a value approximately equal to $-a / z$. This is obviously the wrong answer, if $\operatorname{Re} z>0$. Indeed, from known asymptotic formulas [5, Eq. 13.1.4],

$$
\frac{M^{\prime}(a, b ; z)}{M(a, b ; z)} \sim 1 \quad \text { as }|z| \rightarrow \infty \text { in } \operatorname{Re} z>0 .
$$

What is likely to happen, then, is that the terms $p_{k}$, after the initial descent, begin to increase again, and converge to zero only after reaching some peak values which are sufficiently large so as to contribute to a limit consistent with (2.6). It is only during the "final descent" of the terms $p_{k}$ that the correct limit will be attained (assuming no rounding errors).

The phenomenon of apparent convergence, while prevalent for $\operatorname{Re} z>0$ and $|z|$ large, need not occur if $\operatorname{Re} z<0$, since in this case [5, Eq. 13.1.5]

$$
\frac{M^{\prime}(a, b ; z)}{M(a, b ; z)} \sim-\frac{a}{z} \quad \text { as }|z| \rightarrow \infty \text { in } \operatorname{Re} z<0 .
$$

Nevertheless, we will see in examples that the phenomenon persists if $\pi / 2 \leqslant|\arg z|<\pi$, albeit in a weakened form.

3. The Case of Real $z$. It is instructive to examine in more detail the case of real arguments $z$ and real parameters $a, b$ satisfying $0<a+1$ if $z>0$, and $0<a+1$ $\leqslant b$ if $z<0$.

It will be convenient to introduce the quantities

$$
\sigma_{k}=1+\rho_{k}, \quad k=0,1,2, \ldots
$$

for which the recursion (2.4) gives 


$$
\sigma_{0}=1, \quad \sigma_{k}=\frac{1}{1+a_{k} \sigma_{k-1}}, \quad k=1,2,3, \ldots .
$$

3.1. The Case $z=x>0, a+1>0$. We consider two subcases, (i) $b-x>0$, (ii) $b-x<0$.

In case (i), it follows from (2.2) that $a_{k}>0$ for all $k \geqslant 1$, hence from (3.2) that $0<\sigma_{k}<1$ for all $k \geqslant 1$, and therefore from (3.1) that $-1<\rho_{k}<0$. We see that the terms $p_{k}$ in (2.3) alternate in sign and decrease monotonically in modulus. In fact, since $a_{k} \rightarrow 0$, hence $a_{k} \sigma_{k-1} \longrightarrow 0$ as $k \rightarrow \infty$, we have $\sigma_{k} \rightarrow 1$, and so $\rho_{k} \rightarrow 0$, meaning that the series in (2.3) converges faster than any geometric series. Indeed,

$$
\rho_{k} \sim-\frac{x}{k} \text { as } k \rightarrow \infty,
$$

as is easily verified.

In case (ii), there exists a unique integer $k_{0} \geqslant 1$ such that $x-b<k_{0}<x-b+1$. Therefore, $a_{k_{0}}<0$, while $a_{k}>0$ for all $k \neq k_{0}$. It follows as before that $-1<$ $\rho_{k}<0$ for $k<k_{0}$. If $\sigma_{k_{0}}>0$ (even though $a_{k_{0}}<0$ ), then $-1<\rho_{k}<0$ also for all $k \geqslant k_{0}$, and we have the same alternating and supergeometric convergence behavior as in case (i). If, however, $\sigma_{k_{0}}<0$ (which will be the case if $x$ is large), then $\left|\rho_{k_{0}}\right|>1$. Since $a_{k}>0$ for $k>k_{0}$, the inequality $\left|\rho_{k}\right|>1$ will persist as long as $\sigma_{k}$ remains negative. Eventually, however, $\sigma_{k}$ has to turn positive (the series in (2.3) being convergent), and from this point on, all subsequent $\sigma$ 's remain positive, and the corresponding $\rho$ 's less than one in modulus. Therefore, if $x$ is large, the sequence $\left\{\left|p_{k}\right|\right\}$ initially decreases, then increases, and finally decreases to zero at a supergeometric rate given by (3.3). The "dip-and-peak" effect is more pronounced, the larger $x$, and is what gives rise to the phenomenon of apparent convergence.

3.2. The Case $z=-x<0,0<a+1 \leqslant b$. This time, $a_{k}<0$ for all $k \geqslant 1$. Noting that the function $x(b+x+k-1)^{-1}(b+x+k)^{-1}$ on $0 \leqslant x<\infty$ assumes a unique maximum at $(b+k-1)^{1 / 2}(b+k)^{1 / 2}$, we find that

$$
\left|a_{k}\right| \leqslant \frac{a+k}{(\sqrt{b+k}-1+\sqrt{b+k})^{2}}, \quad k \geqslant 1,
$$

and thus, in particular,

$$
\begin{aligned}
\left|a_{k}\right| & \leqslant \frac{a+k}{2 b+2 k-1+2 \sqrt{b+k-1} \sqrt{b+k}}<\frac{a+k}{2 b+2 k-1+2(b+k-1)} \\
& <\frac{1}{4} \frac{a+k}{b+k-1} .
\end{aligned}
$$

Since $a+1 \leqslant b$, it follows that

$$
a_{k}<0, \quad\left|a_{k}\right|<1 / 4 \quad \text { for all } k \geqslant 1 .
$$

From this, and (3.2), we deduce inductively

$$
1<\sigma_{k}<\frac{2(k+1)}{k+2} \text { for all } k \geqslant 1,
$$

hence, in particular, $0<\rho_{k}<1$ for all $k \geqslant 1$. The series in (2.3) is now a series of 
positive monotonically decreasing terms, and convergence thus monotone and, as before, supergeometric.

\section{Examples.}

4.1. Bessel Functions. We specialize (1.1) to $a=\nu+1 / 2, b=2 \nu+1$, where $\nu \geqslant 1 / 2$, and use $M(\nu+1 / 2,2 \nu+1 ; z)=\Gamma(1+\nu) \exp (1 / 2 z)(1 / 4 z)^{-\nu} I_{\nu}(1 / 2 z)$, together with the differential-difference relation $I_{\nu}^{\prime}(z)=I_{\nu-1}(z)-\nu I_{\nu}(z) / z$, to obtain

$$
\begin{aligned}
& \frac{1}{2} z\left\{\frac{I_{\nu-1}(1 / 2 z)}{I_{\nu}(1 / 2 z)}-\frac{4 \nu}{z}+1\right\} \\
& \quad=\frac{\left(\nu+\frac{1}{2}\right) z}{2 \nu+1-z+} \frac{\left(\nu+\frac{3}{2}\right) z}{2 \nu+2-z+} \frac{\left(\nu+\frac{5}{2}\right) z}{2 \nu+3-z+} \cdots=\frac{\left(\nu+\frac{1}{2}\right) z}{2 \nu+1-z} \sum_{k=0}^{\infty} p_{k} .
\end{aligned}
$$

In Figure 4.1, the moduli of the terms, $\left|p_{k}\right|$, are plotted in function of $k$, for $\nu=1$, and $z=r e^{i \varphi}, r=10,20,40, \varphi=0, \pi / 8,2 \pi / 8, \ldots, \pi$. The behavior of $\left|p_{k}\right|$, when $r$ is fixed and $\varphi$ varies between 0 and $\pi / 2$, is almost identical and is represented by one curve in Figure 4.1. The dependence on $\varphi$ is shown only in the case $r=40$, but is analogous for the other values of $r$.

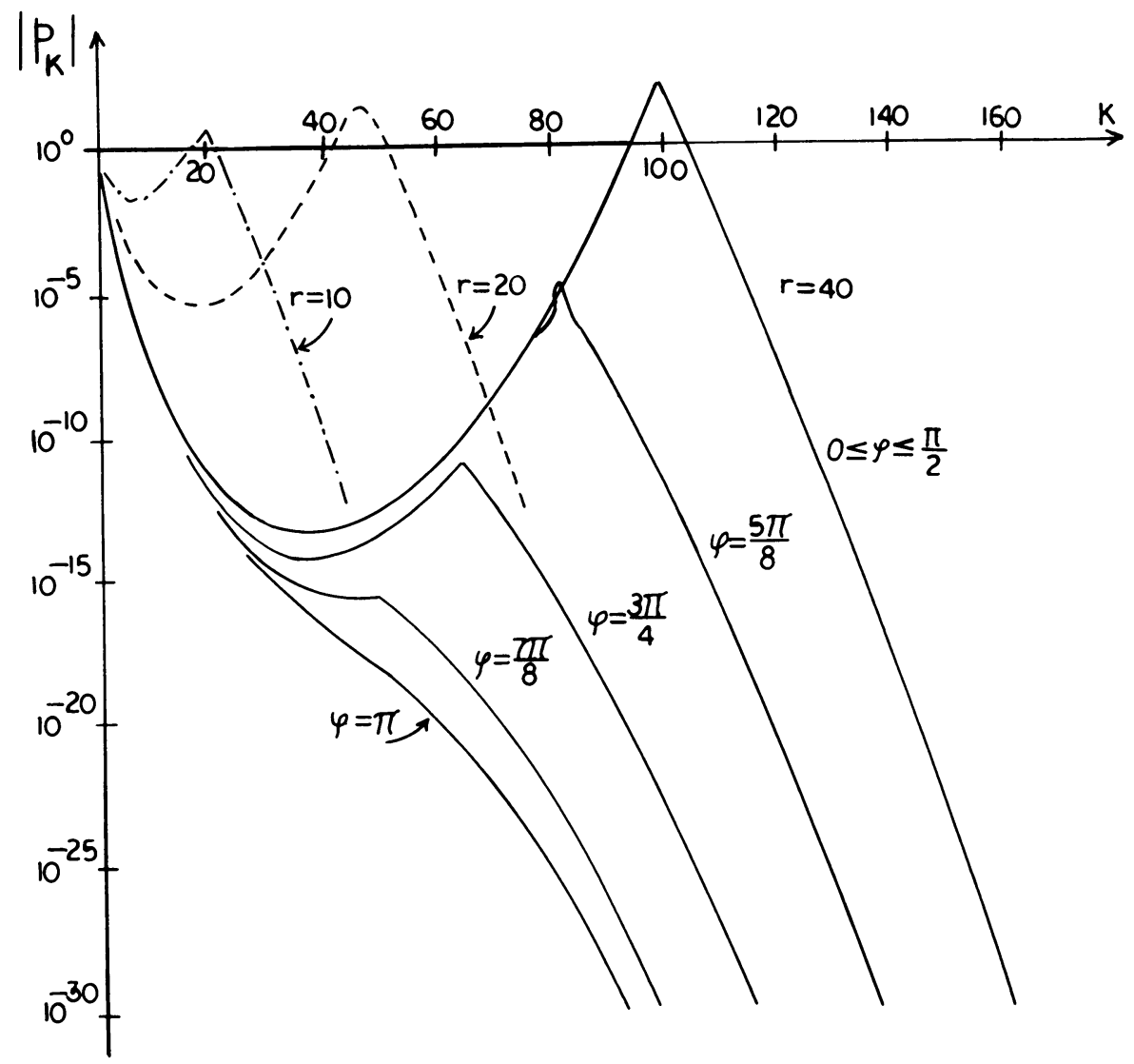

FigURE 4.1

Anomalous convergence of (4.1) for $\nu=1$ and $z=r e^{i \varphi}$ 
It is seen, typically, that the terms $\left|p_{k}\right|$ decrease rapidly at the beginning, then bottom out and rise to a sharp peak, before finally converging to zero. The dip of the curve, and the upswing that follows, are quite substantial if $0 \leqslant \varphi \leqslant \pi / 2$ and $r$ is large, for reasons explained in subsection 3.1, case (ii). As $\varphi$ increases from $\pi / 2$ to $\pi$, the peaking of the curves gradually weakens and finally disappears when $\varphi=\pi$. A similar attenuation takes place upon increasing the value of $\nu$, as is to be expected from the discussion in subsection 3.1, case (i).

The seriousness of the convergence anomaly can be seen, e.g., in the case $\nu=1$, $r=40,0 \leqslant \varphi \leqslant \pi / 2$. If we require ten decimal digit accuracy, we will attain it at about $k=15$ and retain it through about $k=60$, the partial sums in (4.1) all having the same value to ten decimal digits in the range $15 \leqslant k \leqslant 60$. This "apparent limit", of course, is totally incorrect, as the main contribution to the series comes from the few terms around $k=100$. The situation is aggravated by the fact that the numerical process of generating the terms $p_{k}$ is accompanied by a substantial loss of accuracy during the upswing of the curve, amounting to a loss of about 16 digits when $r=40$. A further complication is the apparent lack of warning signals: Known a posteriori error estimates (see, e.g., [3]) either do not apply, or seem to apply only in the region of "final descent".

On the other hand, the convergence behavior of the continued fraction in (4.1) is quite acceptable when $\varphi=\pi$, i.e., $z=-x, x>0$, in which case (4.1) can be given the form

$$
\frac{1}{2} x\left\{\frac{I_{\nu-1}(1 / 2 x)}{I_{\nu}(1 / 2 x)}-\frac{4 v}{x}-1\right\}=\frac{-\left(\nu+\frac{1}{2}\right) x}{2 v+1+x-} \frac{\left(v+\frac{3}{2}\right) x}{2 v+2+x-} \frac{\left(v+\frac{5}{2}\right) x}{2 v+3+x-} \cdots .
$$

Convergence is more rapid the larger $x$ and/or $\nu$. The use of this continued fraction, in combination with Gauss' continued fraction, is further discussed in [2]

4.2. Incomplete Gamma Function. We have $M(a, a+1 ; z)=a(-z)^{-a} \gamma(a,-z)$, where $\gamma(a, \cdot)$ denotes the incomplete gamma function. Noting that

$$
M^{\prime}(a, a+1 ; z)=\frac{a}{a+1} M(a+1, a+2 ; z),
$$

Eq. (1.1) now takes the form

$$
\frac{\gamma(a+1,-z)}{\gamma(a,-z)}=\frac{-a z}{a+1-z+} \frac{(a+1) z}{a+2-z+} \quad \frac{(a+2) z}{a+3-z+} \cdots .
$$

The convergence behavior of (4.3) for $a>0$ appears to be quite analogous to that of (4.1), exhibiting the phenomenon of apparent convergence for $z$ large in the complex plane cut along the negative real axis. Along the negative real axis, we have monotone convergence, if $a>-1$, according to the results of subsection 3.2. In this case,

$$
\frac{\gamma(a+1, x)}{\gamma(a, x)}=\frac{a x}{a+1+x^{-}} \frac{(a+1) x}{a+2+x^{-}} \quad \frac{(a+2) x}{a+3+x^{-}} \cdots, \quad x>0 .
$$

We may combine this with $\gamma(a+1, x)=a \gamma(a, x)-x^{a} e^{-x}$ to obtain

$$
x^{-a} e^{x} \gamma(a, x)=\frac{1}{a^{-}} \quad \frac{a x}{a+1+x^{-}} \frac{(a+1) x}{a+2+x^{-}} \quad \frac{(a+2) x}{a+3+x^{-}} \cdots, \quad x>0 .
$$


The use of this continued fraction, in combination with other methods, to evaluate incomplete gamma functions is discussed in [1].

Department of Computer Sciences

Purdue University

Lafayette, Indiana 47907

1. W. GAUTSCHI, "An evaluation procedure for incomplete gamma functions," $A C M$ Trans. Mathematical Software. (To appear.)

2. W. GAUTSCHI \& J. SLAVIK, "On the computation of modified Bessel function ratios," Math. Comp. (To appear.)

3. W. B. JONES, "Analysis of truncation error of approximations based on the Padé table and continued fractions," Rocky Mountain J. Math., v. 4, 1974, pp. 241-250. MR 49 \#6551.

4. O. PERRON, Die Lehre von den Kettenbrüchen, Vol. II, 3rd ed., Teubner, Stuttgart, 1957. MR 19, 25.

5. L. J. SLATER, "Confluent hypergeometric functions," Handbook of Mathematical Functions with Formulas, Graphs, and Mathematical Tables (M. Abramowitz \& I. A. Stegun, Editors), Nat. Bur. Standards, Appl. Math. Ser., no. 55, Superintendent of Documents, U. S. Government Printing Office, Washington, D. C., 1964, pp. 503-535. MR 29 \#4914.

6. H. S. WALL, Analytic Theory of Continued Fractions, Van Nostrand, New York, 1948; reprint, Chelsea, New York, 1967. MR 10, 32. 